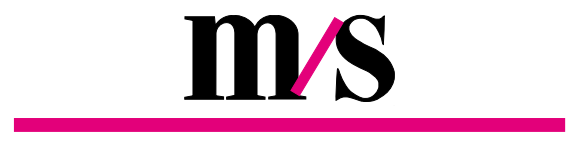

médecine/sciences $1999 ; 15: 775-7$

\title{
PHYSIOPATHOLOGIE PULMONAIRE : \\ DE LA RECHERCHE FONDAMENTALE AUX AVANCÉES THÉRAPEUTIQUES
}

es maladies bronchiques asthme, bronchite chronique et emphysème représentent un véritable problème de santé publique. Ces affections touchent, en France, environ 6 millions de personnes et, comme dans tous les pays industrialisés, elles sont en augmentation constante. Les raisons à l'origine de l'augmentation de la prévalence de ces maladies ne sont pas parfaitement élucidées, mais il est clair que des facteurs liés à l'environnement y jouent un rôle essentiel.

La connaissance des mécanismes physiopathologiques de ces maladies a permis, ces dernières années, des avancées thérapeutiques majeures. L'utilisation des corticoïdes inhalés qui ont radicalement transformé le quotidien des patients asthmatiques - en est une excellente illustration. En effet, à l'aube du $\mathrm{XXI}^{\mathrm{e}}$ siècle, la recherche en physiologie et en physiopathologie pulmonaire a considérablement progressé grâce aux importantes avancées de l'immunologie, de la biochimie et de la biologie cellulaire et moléculaire. Néanmoins, si la physiologie respiratoire classique, représentée par la mécanique ventilatoire, fait désormais partie de l'exploration clinique de routine, elle reste à l'origine des progrès récents dans des domaines tels que la ventilation artificielle qui en consti- tue une des applications thérapeutiques essentielles. Cette dernière, qui représente l'outil thérapeutique majeur de l'insuffisance respiratoire aiguë, constitue une excellente illustration des progrès réalisés grâce à l'apport de la physiologie classique et de la biologie moderne. En effet, les avancées dans le domaine de la ventilation artificielle proviennent à la fois de travaux concernant les mécanismes de contrôle central de la ventilation et de l'étude de la mécanique respiratoire ainsi que des effets des pressions et des volumes d'insufflation sur les structures alvéolaires et les mécanismes réglant la réabsorption des liquides au sein de l'alvéole [1] (voir l'article de G. Saumon, p. 778 de ce numéro). Ainsi, 10 ans après les études expérimentales chez l'animal montrant les effets délétères des hauts volumes d'insufflation sur les stuctures alvéolaires, les modalités de ventilation des patients ont été modifiées suivant ces résultats en diminuant le volume courant du ventilateur. Une meilleure compréhension de l'interaction ventilateurpatient - tant au niveau de la mécanique respiratoire que sur celui de la mécanique cellulaire ou au plan des réactions inflammatoires induites ou inhibées par la ventilation - ont ainsi permis de réduire la mortalité dans des affections telles que le syndrome de détresse respira- 
toire aiguë ou les poussées de décompensation de broncho-pneumopathie chronique obstructive. La modification ventilatoire la plus spectaculaire proposée ces dernières années, et qui a permis de diminuer considérablement les facteurs de morbidité liés à la ventilation assistée, est la ventilation dite non invasive [2] (voir l'article de L. Brochard, p. 798 de ce numéro) délivrée par un masque facial ou nasal dans le traitement des décompensations aiguës de brochopneumopathie chronique obstructive (BPCO).

L'insuffisance respiratoire aiguë représente le stade d'évolution ultime de nombreuses maladies pulmonaires telles que les BPCO. Il est clair que la prise en charge thérapeutique doit avoir pour objectif d'éviter cette évolution.

L'asthme a longtemps été considéré comme la résultante d'anomalies du muscle lisse des voies aériennes dont la contraction exagérée, secondaire à différents stimulus (physique, pharmacologiques, allergéniques...) est responsable d'un bronchospasme. Depuis 20 ans, les méthodes d'investigation bronchique par tube souple ont permis de mettre en évidence une composante inflammatoire de l'asthme. L'asthme est actuellement défini comme un "syndrome chronique inflammatoire des voies aériennes, plus particulièrement des bronches, dans lequel de nombreux types cellulaires, dont les éosinophiles et les lymphocytes $\mathrm{T}$, jouent un rôle " [3] (voir l'article de J. Bousquet et al., p. 823 de ce numéro). Cette même définition décrit l'inflammation comme le phénomène responsable des symptômes de la maladie, tels que les crises de dyspnée paroxystique sifflante, d'essoufflement, d'oppression thoracique et de toux. Cette inflammation, observée au niveau de la muqueuse bronchique de sujets asthmatiques, est constituée par la présence d'éosinophiles et est généralement associée à une infiltration de lymphocytes $\mathrm{T}$ totaux ou de phénotype $\mathrm{CD}^{+}, \mathrm{CD}^{+}$, gd, de cellules $\mathrm{B}$, de macrophages, de cellules dendritiques, de mastocytes, de basophiles, de neutrophiles et de plaquettes sanguines [4]. L'activation de été clairement démontrée. Par ailleurs, les lymphocytes $\mathrm{T}$ sanguins et bronchiques de patients asthmatiques présentent une polarisation vers le phénotype $T$ helper 2 (TH2), comme en témoigne l'expression de l'ARNm codant pour l'interleukine-4 (IL-4), l'IL-5 et l'IL-13 associée à ces cellules [5]. Des molécules possédant des propriétés anti-inflammatoires plus ciblés que les glucocorticoides, telles que les antagonistes des récepteurs des leucotriènes [6] sont actuellement disponibles alors que d'autres, comme les anticorps monoclonaux anti-IgE, sont actuellement en phase III. De même, des thérapies visant à inhiber efficacement et plus sélectivement l'inflammation éosinophilique pourraient représenter une perspective intéressante dans le traitement de l'asthme. De nombreux facteurs, comme l'IL-5, sont capables de favoriser l'éosinophilie sanguine et tissulaire et de contribuer à sa persistance. Cette cytokine, en effet, constitue le stimulus le plus sélectif et l'un des plus puissants car agissant sur la plupart des fonctions et à toutes les étapes de la vie de ces cellules. De ce fait, elle représente une cible thérapeutique potentielle. Ainsi, des essais cliniques sont actuellement en cours dans le but de démontrer l'efficacité d'un anticorps humanisé dirigé contre l'IL-5 dans le traitement de l'asthme modéré [7].

En ce qui concerne les maladies inflammatoires chroniques, dont l'asthme, plusieurs auteurs s'accordent à émettre l'hypothèse selon laquelle un déficit apoptotique des granulocytes, des monocytes-macrophages ou des lymphocytes $\mathrm{T}$ pourrait également jouer un rôle dans le développement et la persistance de l'inflammation tissulaire [8]. Ce déficit pourrait être dû : (1) à une surexpression et à une libération incontrôlée de facteurs de survie, tels que l'IL-5 et le GM-CSF (granulocyte-macrophage colony stimulating factor), par les cellules inflammatoires, les cellules résidentes tissulaires ou les lymphocytes $\mathrm{T}$ qui sont activés dans la plupart de ces maladies et/ou (2) à une modification de l'expression ou de la fonction des molécules impliquées dans l'apoptose et/ou dans la phagocytose des granulocytes et des lym- phocytes $\mathrm{T}$, telles que les protéines de la famille de Bcl-2, Fas et Fas-L. Ainsi, l'apoptose peut jouer un rôle à la fois physiologique et pathologique. Dans le cas de l'inflammation, la mort par apoptose semble être un processus important lors de la résolution normale de la réaction inflammatoire. Un déficit ou un excès d'apoptose pourrait être impliqué dans le développement de l'inflammation aiguë et, ainsi, pourrait favoriser l'inflammation chronique, caractéristique de l'asthme. La connaissance des mécanismes contribuant à l'élimination des éosinophiles par apoptose et des molécules mises en jeu dans les voies de signalisation qui règlent ce phénomène permettront de cerner les étapes clés impliquées dans le démarrage et le maintien de l'éosinophilie sanguine et tissulaire lors des processus atopiques. Cela ouvrirait des perspectives thérapeutiques nouvelles dans le traitement de l'asthme.

Contrastant avec les avancées considérables dans la connaissance des mécanismes physiopathologiques et, par voie de conséquence, dans le traitement de l'asthme, peu de progrès ont été réalisés en 20 ans dans la prise en charge thérapeutique des bronchopathies chroniques obstructives non réversibles. Les raisons en sont multiples : (1) l'hétérogénéité des maladies regroupées sous le terme de BPCO (bronchite chronique, emphysème centro- et panlobulaire, bronchiolite) ; (2) le caractère tardif des manifestations cliniques ; (3) la difficulté de recueillir des cellules du poumon profond ; (4) la méconnaissance, dans le poumon distal, des processus inflammatoires, notamment des cellules impliquées et de leur rôle respectif; (5) la nature du déséquilibre protéase-antiprotéase aboutissant à la destruction des parois alvéolaires ainsi que les cibles de ces protéases (élastine?, collagène ?) dans les différents types d'emphysème ; (6) l'ignorance presque totale des processus de réparation et de leurs anomalies notamment des fibres de collagène.

Il faut cependant souligner l'évolution récente des concepts concernant les mécanismes physiopathologiques de l'emphysème. En effet, le rôle consi- 
déré comme fondamental des polynucléaires neutrophiles et de l'élastase dans la destruction des parois alvéolaires est largement remis en cause. Actuellement, l'importance des macrophages alvéolaires dans la genèse de l'emphysème apparaît comme beaucoup plus important. Cela a été bien confirmé sur des modèles de souris génétiquement modifiées. Ainsi, des souris surexprimant le gène de la collagénase interstitielle, une protéase n'ayant pas de propriété élastolytique, développent un emphysème pulmonaire morphologiquement proche de l'emphysème humain induit par le tabac [9] (voir l'article de H. Mal et al., p. 833 de ce numéro). De même, des souris, dont le gène codant pour l'élastase macrophagique a été invalidé, ne développent pas d'emphysème lorsqu'elles sont exposées à la fumée de tabac [10]. Ces deux études mettent en exergue le rôle du macrophage dans la genèse de l'emphysème et relativisent le rôle du polynucléaire neutrophile classiquement considéré comme la cellule clé de la physiopathologie de l'emphysème pulmonaire. Un travail très récent est encore venu conforter le rôle d'un dysfonctionnement du macrophage alvéolaire dans la genèse de l'emphysème et plus particulièrement celui des macrophages dont le cytoplasme contient des inclusions lipidiques qui s'accumulent dans les alvéoles. En effet, des souris dont le gène codant pour l'apoprotéine D du surfactant a été invalidé, développent un emphysème pulmonaire [11]. La prise en charge thérapeutique du fait de la méconnaissance relative à l'échelon cellulaire des mécanismes physiopathologiques conduisant à l'emphysème - reste donc pour l'instant palliative. Elle est actuellement essentiellement chirurgicale avec deux options possibles, la greffe pul- monaire (actuellement mono- ou bipulmonaire) et la chirurgie de réduction de volume.

Les traitements médicaux restent essentiellement symptomatiques et visent à améliorer la dyspnée. Dans ce cadre, il faut remarquer que si les possibilités d'améliorer ce symptôme, qui est à l'origine de l'essentiel du handicap respiratoire, restent limitées, les techniques d'imagerie fonctionnelle modernes telles que la tomographie par émission de positons ont permis de localiser les structures cérébrales impliquées dans la sensation de dyspnée. Il pourrait donc être envisageable dans le futur de moduler pharmacologiquement l'activité de ces stuctures, ce qui pourrait s'accompagner d'une diminution de la sensation de dyspnée chez les patients présentant une insuffisance respiratoire.

De la réanimation respiratoire au traitement de l'inflammation des voies aériennes, les approches physiopathologiques des maladies pulmonaires sont multiples, mais très complémentaires. L'approche des processus inflammatoires dans le poumon ne peut être dissociée de l'approche physiologique représentée par la mécanique respiratoire. Des avancées spectaculaires ont été réalisées au cours de ces 20 dernières années dans des affections telles que l'asthme tant dans la compréhension des mécanismes de l'inflammation bronchique, caractéristique de cette maladie, que de sa prise en charge thérapeutique. La réanimation respiratoire s'est enrichie de nouvelles techniques à l'origine de la diminution de la mortalité de l'insuffisance respiratoire aiguë. Des progrès majeurs se profilent à l'aube du $\mathrm{XXI}^{\mathrm{e}}$ siècle dans la connaissance de maladies telles que l'emphysème

\section{RÉFÉRENCES}

1. Dreyfus D, Soler P, Saumon G. Mechanichal ventilation-induced pulmonary edema. Interaction with previous lung alterations. Am J Respir Crit Care Med 1995 ; 151 : 1568-75.

2. Brochard L, Isabey D, Piquet J, et al. Reversal of acute exacerbation of chronic obstructive lung disease by inspiratory assistance with a face mask. N Engl J Med $1990 ; 323$ : 1523-30.

3. International consensus report on diagnosis and treatment of asthma. Eur Respir J $1992 ; 5$ : 601-41.

4. Djukanovic R. Bronchial biopsies. In : Busse WW, Holgate ST, eds. Asthma and rhinitis. Boston : Blackwell Scientific Publications, 1995 : 118-29.

5. Robinson DS, Hamid Q, Ying S, et al. Predominant $\mathrm{T}_{\mathrm{H}}$ 2-like bronchoalveolar T-lymphocyte population in atopic asthma. NEngl JMed 1992 ; $326: 298-304$.

6. Drazen JM, Israel E, O'Byrne P. Treatment of asthma with drugs modifying the leukotriene pathway. NEngl J Med 1999 ; 340 : 197 206.

7. Egan RW, Athwahl D, Chou CC, et al. Inhibition of pulmonary eosinophilia and hyperreactivity by antibodies to interleukin- 5 . Int Arch Allerg Immunol 1995 ; 107 : 321-2.

8. Simon HU, Blaser K. Inhibition of programmed eosinophil death : a key pathogenic event for eosinophilia? Immunol Today $1995 ; 16: 53-5$.

9. D'Armiento J, Dalal SS, Okada Y, et al. Collagenase expression in the lungs of transgenic mice causes pulmonary empysema. Cell $1992 ; 71: 955-61$.

10. Hautamaki RD, Kobayashi DK, Senior RM, Shapiro SD. Requirement for macrophage elastase for cigarette smoke-induced emphysema in mice. Science 1997 ; 277 : 20024.

11. Whitsett JA, Wert SE, Jones T, Fisher J, Korfhagen TR. Spontaneous emphysema in $S P-D$ gene targeted mice. Am J Respir Crit Care Med 1999 ; 159 : A729.

\section{TIRÉS À PART}

M. Aubier.

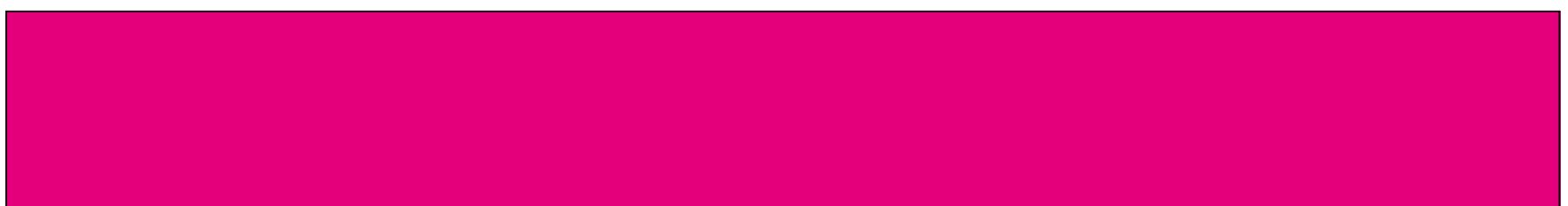

